\title{
Epidemiología del abuso de alcohol entre la población inmigrante en España
}

\section{Epidemiology of Alcohol Abuse Among Spanish Immigrant Populations}

\author{
Luis Sordo ${ }^{* * * * * * *}$, Blanca Iciar Indave*, Jose Pulido*,***, Gemma Molist*****, María Elena Rosales- \\ Statkus ${ }^{* * * * *}$, Mónica Ruíz-García ${ }^{*, * * *}$, Gregorio Barrio***** \\ * Centro Nacional de Epidemiología. Instituto de Salud Carlos III. Madrid \\ ** Departamento de Medicina Preventiva y Salud Pública. Facultad de Medicina. Universidad Complutense de Madrid \\ *** CIBER Epidemiología y Salud Pública (CIBERESP) \\ ***** Escuela Nacional de Sanidad. Instituto de Salud Carlos III. Madrid
}

\section{Resumen}

A pesar del gran incremento de la población inmigrante en los últimos años, su uso de alcohol está poco estudiado. Se describe la epidemiología del consumo de riesgo de alcohol en la población inmigrante residente en España, frente a la nativa. Se emplearon datos de 22188 respondentes a la Encuesta Europea de Salud de 2009, de los que 3162 eran extranjeros. Como indicadores de consumo problemático se usó la prevalencia de consumo excesivo promedio y el consumo excesivo episódico. Se realizaron análisis descriptivo de la población, determinación de zonas de procedencia con mayor consumo de alcohol y factores relacionados para cada tipo de consumo separando población inmigrante de autóctona. El perfil sociodemográfico del inmigrante fue heterogéneo, aunque predominantemente de entre 35 y 54 años, que vive en familia y trabaja. Se consideraron bebedores excesivos promedio al $3,4 \%$ de los inmigrantes por el $3,2 \%$ de los nativos, y bebedores excesivos episódicos en el último año el 8,9\% frente al $10 \%$. Los inmigrantes procedentes de Europa del Norte y del Oeste, y América latina, países andinos, fueron aquellos que presentaron mayores razones de prevalencia de bebedores de riesgo que la población nativa. Por el contrario, proceder de África fue un factor protector. De los factores relacionados con un mayor consumo, destaca el desempleo, siendo más relevante en la población inmigrante. El consumo excesivo de alcohol en inmigrantes es muy heterogéneo, debiendo adecuarse las intervenciones sobre el mismo a su situación social, diferentes entornos y áreas de procedencia.

Palabras clave: Inmigración, Uso de alcohol, Dependencia de alcohol, Consumo excesivo de alcohol.

\begin{abstract}
In recent years, the immigrant population has substantially increased in Spain. However, there is a lack of information in the knowledge of alcohol abuse among Spanish immigrants. We describe the epidemiology of alcohol abuse among foreign-born immigrants versus Spanish natives. We carried out a cross-sectional study that uses data from the European Survey of Health on the General Population of Spain of 2009. A sample of 22,188 subjects was analyzed (of whom, 3,162 were foreign). Proxies of problematic alcohol consumption were the prevalence of excessive average consumption and the prevalence of excessive episodic consumption (binge drinking). Descriptive analysis of the population, determination of area of origin with major alcohol consumption and related factors for each kind of consumption, separating immigrant and native population, were performed. The immigrant profile was heterogeneous, though predominantly aged between 35 and 54, and were living with their family and working. $3.4 \%$ of immigrants and $3.2 \%$ of natives were considered excessive drinkers; $8.9 \%$ and $10 \%$, respectively, reported binge drinking in the last year. Immigrants from Northern and Western Europe, and Latin America, Andean countries had significantly a higher report of frequent alcohol consumption and/or binge drinking compared to native. On the contrary, born in Africa was a protective factor. Unemployment was the most relevant related factor, being more important in the immigrant population. The excessive alcohol consumption in immigrants is dissimilar; the interventions must be adapted to their social situation, environments and areas of origin. Key words: Immigrant status; Alcohol abuse; Alcohol dependence; Excessive drinking.
\end{abstract}


$\mathrm{E}$

l consumo de alcohol es uno de los principales factores de riesgo de enfermedad y muerte prematura en todo el mundo (World Health Organization (WHO), 2014; Lim et al., 2012). Se relaciona con riesgo de dependencia, cirrosis hepática, accidentabilidad y otros importantes problemas de salud (Anderson P, 2012). Sin embargo, a pesar de la enorme carga de enfermedad asociada al consumo de alcohol, las intervenciones de salud pública para reducir su impacto siguen siendo insuficientes (WHO, 2014).

Muchas consecuencias del consumo problemático de alcohol dependen de su patrón de cantidad o volumen, y de la frecuencia con la que se haga. Básicamente se diferencian dos tipos de consumo de riesgo de alcohol (CRA): el consumo excesivo promedio semanal elevado (CEP) (WHO, 2000), y el consumo excesivo episódico (CEE), conocido también en la literatura científica como bingedrinking, que implica un consumo muy elevado de alcohol en periodos muy cortos (Valencia-Martin, Galan, y Rodriguez-Artalejo, 2007). Aunque los dos tipos de consumo tienen consecuencias comunes, como hipertensión, pancreatitis o patología hepática, el CEE se relaciona más frecuentemente con accidentes de tráfico y episodios de violencia (WHO, 2014).

Los determinantes del consumo de alcohol son múltiples; bien individuales, como el nivel educativo o aspectos genéticos (Martinez-Hernaez, Mari-Klose, Julia, Escapa, y Mari-Klose, 2012), bien grupales o contextuales. Entre estos últimos, destacan el desarrollo económico, la cultura, la disponibilidad de alcohol o la efectividad de las políticas sociales o sanitarias de cada país (WHO, 2010; Babor et al., 2007; Rehm, Rehm, Shield, Gmel y Gual, 2013). Estos factores determinan las diferencias en el nivel de consumo entre grupos poblacionales o naciones (Anderson P, 2012). En este sentido, la enorme movilidad geográfica entre países observada en los últimos años, plantea nuevos retos sociales y sanitarios.

A menudo hay desigualdades en el nivel y los determinantes de salud entre el país de procedencia de la población inmigrante y el país de destino. Estas diferencias tienen efectos variables sobre la salud de inmigrantes y nativos (MacPherson y Gushulak, 2001). Se suele asumir un peor nivel de salud en los inmigrantes frente a los nativos, pero está bien documentado el "efecto del inmigrante sano": los recién llegados tienen por término medio mejor salud que sus pares en el país de origen e incluso mejor que sus pares del país receptor (De Maio, 2010). Sin embargo, esto no tiene que ser necesariamente así para los determinantes de la salud más mudables como son los patrones de uso de alcohol (Gutmann, 1999).

La epidemiología del consumo de alcohol entre los inmigrantes en España ha sido escasamente estudiada a pesar del importante incremento de esta población. En los últimos años la población no autóctona se ha cuadruplicado hasta constituir en la actualidad el $10 \%$ de la población residen- te en España (Observatorio permanente de la inmigración [OPI], 2006). Algunos estudios indican que los inmigrantes tienen una prevalencia de consumo de alcohol menor al de la población nativa (Carrasco-Garrido, de Miguel, Barrera, y Jimenez-Garcia, 2007). Pero igualmente señalan que esta no es homogénea y que depende del país de procedencia (Marsiglia, Kulis, Luengo, Nieri, y Villar, 2008). Respecto a sus factores de riesgo, estudios realizados fuera de España demuestran que estos son heterogéneos. Los inmigrantes van adquiriendo los patrones del país de origen, al tiempo que su consumo de alcohol puede verse incrementado por procesos desadaptativos, como el proceso de aculturización o el estrés migratorio, relacionados con el nuevo país receptor (Mills y Caetano, 2012; Zamboanga, Schwartz, Jarvis, y Van, 2009).

El objetivo del presente estudio es determinar, separadamente para población autóctona y para inmigrantes en España, la prevalencia de consumo excesivo promedio y episódico de alcohol, así como los factores sociodemográficos asociados a estos indicadores.

\section{Método}

\section{Diseño, población y recolección de datos.}

Los datos analizados en este estudio proceden de la Encuesta Europea de Salud 2009, enmarcada en la propuesta de la Comisión Europea de crear un sistema europeo de información sanitaria. Dicha encuesta tiene como objetivo proporcionar información sobre la salud de la población residente en España de manera armonizada y comparable con el resto de Europa, con la finalidad de planificar y evaluar las actuaciones en materia sanitaria (Instituto Nacional de Estadística (INE), 2009).

La encuesta se dirigió al conjunto de personas residentes en viviendas familiares principales de toda España. Los participantes se seleccionaron mediante un muestreo trietápico con estratificación de las unidades de primera etapa. Estas fueron las secciones censales existentes en enero de 2008, cuya selección se realizó con probabilidad proporcional a su tamaño. Las unidades de segunda etapa fueron los hogares familiares principales de las secciones seleccionadas y se obtuvieron del Padrón Continuo de Habitantes. La selección de hogares se realizó mediante un muestreo sistemático con arranque aleatorio y probabilidades iguales de selección para cada hogar de la sección. Finalmente, dentro de cada hogar se seleccionó (con la misma probabilidad) a una persona adulta (16 ó más años) de las que lo componían. Para facilitar estimaciones con un grado aceptable de fiabilidad a nivel nacional y de comunidad autónoma, se seleccionaron 1927 secciones censales y 12 hogares por sección. La recogida de datos se realizó entre abril de 2009 y marzo de 2010 mediante entrevista personal asistida por ordenador (CAPI), excepto las preguntas sobre "gasto de su propio bolsillo", y consumo de tabaco, alcohol y drogas, 
que fueron autoadministradas con el fin de preservar aún más la confidencialidad y facilitar la veracidad en las respuestas. El presente estudio se realizó sobre todos los participantes en la encuesta salvo aquellos en que se desconocía el país de origen y/o el consumo de alcohol $(n=22.188)$.

\section{Variables y Definiciones}

De las cinco secciones del cuestionario, para este estudio se analizaron los módulos sobre características sociodemográficas, estado y determinantes de salud. En cuanto a las variables de interés, se consideró "inmigrante" aquella persona cuyo país de origen (de nacimiento) no fuera España. Ante la heterogeneidad de las procedencias y la imposibilidad de analizar los datos país por país, estos se agruparon en áreas de origen siguiendo la clasificación de las Naciones Unidas (WHO, 2006): Europa (subdividida en Norte, Sur, Este y Oeste); América Latina (subdividida en Cono Sur y Región Andina); Centroamérica, Caribe y México; África; otros países y España. Las variables dependientes fueron: el consumo excesivo promedio, definido como el consumo promedio en los últimos 12 meses $>40 \mathrm{~g}$ / día (hombres) o $>20 \mathrm{~g}$ /día (mujeres) de alcohol puro, y el consumo excesivo episódico, definido como el consumo al menos una vez durante los últimos 12 meses de seis bebidas alcohólicas o más en la misma ocasión.

Las variables independientes analizadas fueron: sexo, edad (16-34 años, 35-54 y 55 o más), zona de residencia en España (Sur: Andalucía, Murcia, Canarias, Ceuta y Melilla; Este: Comunidad Valenciana, Cataluña y Baleares; Centro: Madrid, Castilla la Mancha y Extremadura; y Norte), tamaño del municipio de residencia, convivencia (solo/a, en pareja y/o con hijos, y otros), nivel de estudios, situación laboral, estado de salud percibido, padecimiento de ansiedad o depresión autorreferidas en últimos 12 meses, exposición a violencia o vandalismo, fumar y consumir cannabis, cocaína, anfetaminas, éxtasis o similares en los 12 últimos meses.

\section{Análisis}

Primero se realizó un análisis descriptivo de las características de la muestra, estratificando según área de origen. Las relaciones entre las diferentes áreas (incluyendo origen español) y entre origen extranjero y autóctono fueron analizados a través de correlaciones de Chi cuadrado (Tabla 1). Después, para evaluar la influencia del área origen sobre los indicadores de consumo de alcohol (CEP y CEE), se ajustaron modelos de regresión de Poisson con varianza robusta, obteniendo razones de prevalencia ajustadas ( $\mathrm{RPa})$ para cada área de origen en relación a los autóctonos y los correspondientes intervalos de confianza al 95\% (IC95\%) (Spiegelman y Hertzmark, 2005). Las variables de ajuste que se incluyeron para cada uno de los modelos (CEP y CEE) fueron aquellas que mostraran $\mathrm{p}<0,10$ en el análisis bivariado con el global de la muestra. Finalmente, separadamente para inmigrantes y autóctonos, se identificaron los factores relacionados con cada uno de los indicadores de consumo de alcohol considerados, utilizando de nuevo modelos de regresión de Poisson con varianza robusta y la $\mathrm{RPa}$ como medida del efecto. Las variables independientes incluidas en cada uno de los modelos fueron aquellas que mostraran una $\mathrm{p}<0,05$ en los análisis bivariantes previos. En los modelos de regresión para muestras de inmigrantes, se ajustó adicionalmente por área de origen.

\section{Resultados}

\section{Características de la muestra}

Los inmigrantes que viven en España, analizados en conjunto, son sobre todo jóvenes de 16-34 años, que residen en el este de España, en poblaciones grandes, con su familia, que trabajan y poseen estudios secundarios; aproximadamente la mitad son mujeres. Su estado de salud es bueno o muy bueno, con una prevalencia de depresión autorrefererida en el último año menor que en los autóctonos (55,5\% y $10,1 \%$ respectivamente) y están poco expuestos a la violencia. Si comparamos este perfil con el de los autóctonos, destaca su baja edad (16,1\% tenían más de 55 años frente a $36,1 \%$ en autóctonos) y un mejor nivel de estudios $(22,6 \%$ tenían estudios universitarios frente a $11,7 \%$ en autóctonos). Estratificando por área de origen, se observan características más heterogéneas, siendo las áreas que más se alejan del perfil promedio descrito: Europa del Norte -donde predominó la población mayor de $55(53,4 \%)$, la residencia en poblaciones de menos de 10.000 habitantes y la situación laboral no activa (52,9\%)-y África -donde predominaron los inmigrantes únicamente con estudios primarios $(50,8 \%)$.

\section{Disparidades en el consumo de alcohol entre nati- vos e inmigrantes de distintas procedencias}

La prevalencia de consumo excesivo promedio (CEP) de alcohol en inmigrantes y nativos fue $3,4 \%$ y $3,2 \%$, respectivamente, mientras que la prevalencia de consumo excesivo episódico (CEE) fue 8,9\% en inmigrantes frente a 10,0\% en nativos. Las diferencias en estas prevalencias según área de origen fueron notables, variando la primera entre 7,0\% para Europa de Norte y 0,5\% para África (si bien para esta área tan sólo dos personas refirieron CEP), y la segunda entre $15,0 \%$ y $2,7 \%$ en las mismas áreas de origen (Tabla 1 ).

Ajustando por los factores confusores identificados en el análisis bivariante, los inmigrantes procedentes de Europa del Norte y del Oeste fueron los que presentaron mayor riesgo de CEP que los autóctonos ( $\mathrm{RPa}=2,28$; IC95\% 1,29-4,05 y $\mathrm{RPa}=2,12$; IC95\% 1,29-3,49, respectivamente), mientras que los procedentes de África presentaron un riesgo menor $(\mathrm{RPa}=0,16$; IC95\% 0,04-0,67). En lo relativo al CEE, las áreas de procedencia con un riesgo significativamente superior al de los nativos fueron Europa del Norte ( $\mathrm{RPa}=1,81$ IC95\% 1,25-2,62) y Región Andina ( $\mathrm{RPa}=1,25 \mathrm{IC} 95 \%$ 1,03-1,5); y aquellas con un riesgo significativamente menor África 


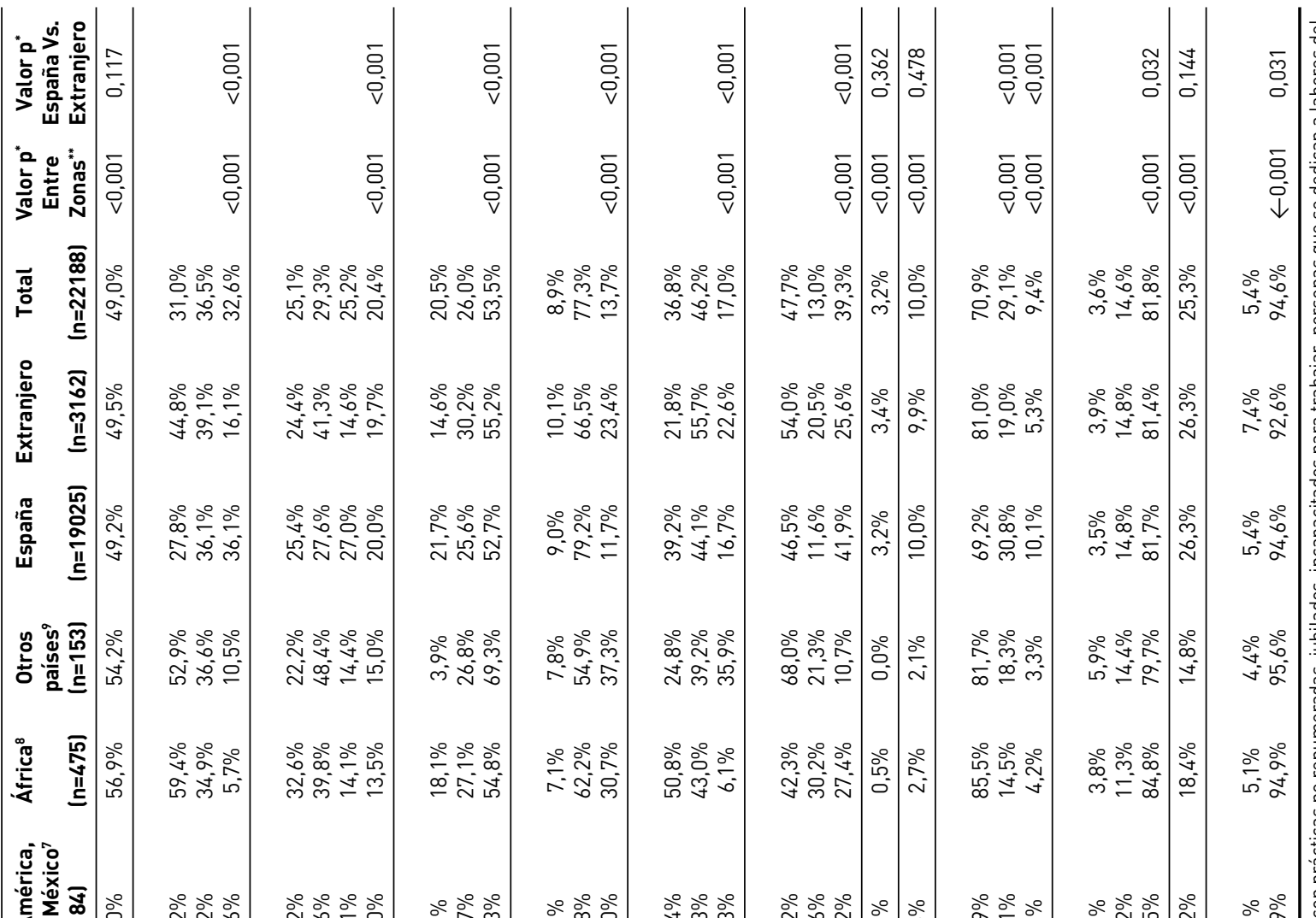

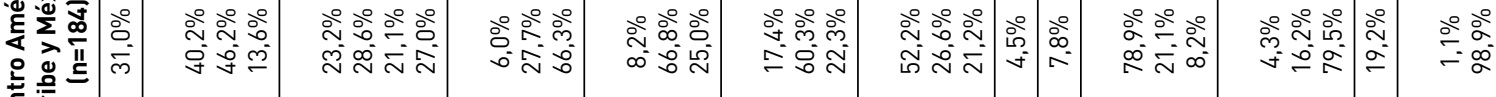
נֶ

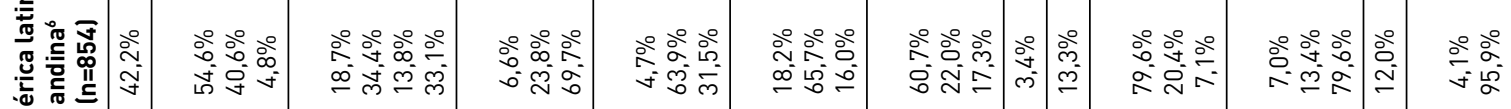
$\stackrel{\oplus}{\mathrm{g}}$

똔

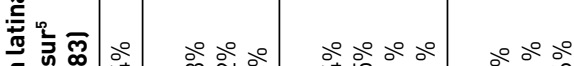
密

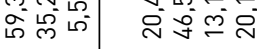

ธ⿻一𠃋十

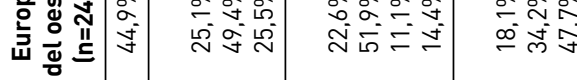

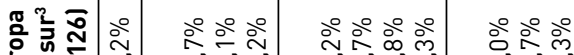

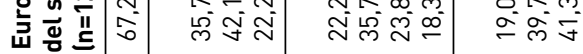

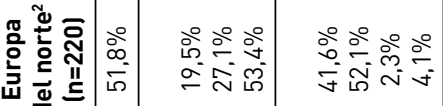
品

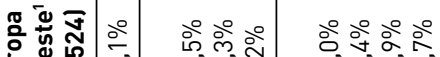

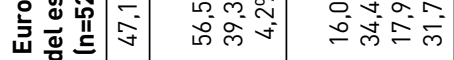

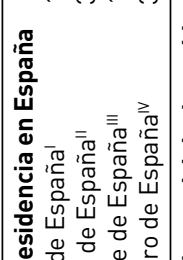

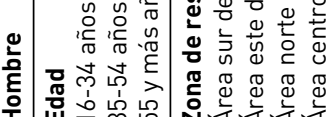

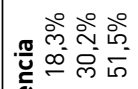

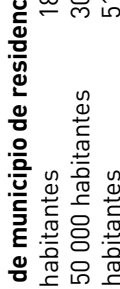

웅요 $\circ$ $\sim+0+\stackrel{\infty}{\sim}$

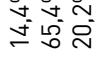

ㅇํㅇำ ㅇำ $\because$ న⿺辶

ㅇํㅇㅇำ นิน

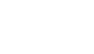

西 눈

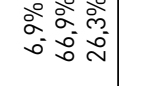

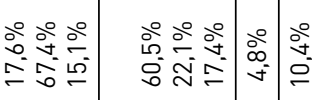

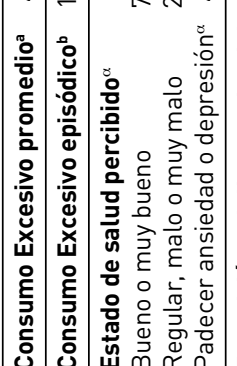

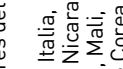
$\frac{\pi}{4} \cdot \frac{\pi}{x} \cdot \frac{\pi}{\frac{\pi}{2}} \frac{0}{0}$

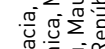

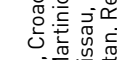
.

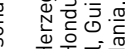
每

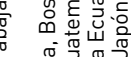
ए人)

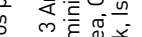

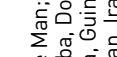
vin ज泡

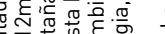

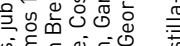

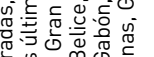

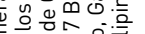

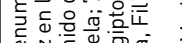

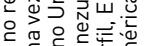
作

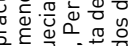
ᄃ

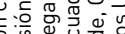

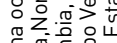
年

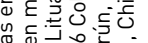

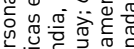

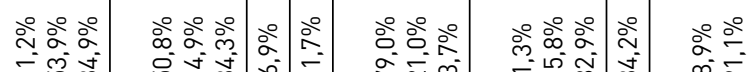

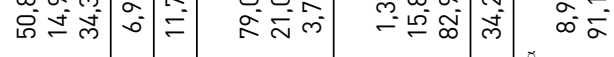

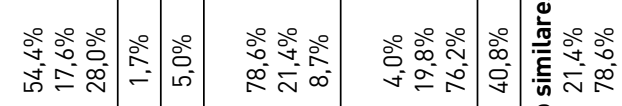
○

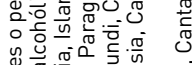

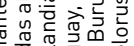

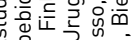
每. ه일

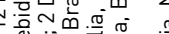

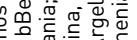

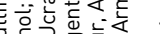

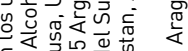

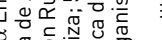

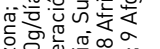

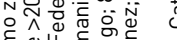

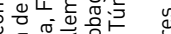
范

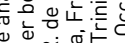

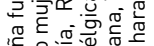

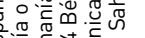
중.

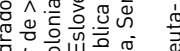

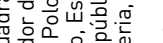

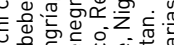

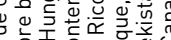

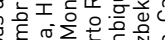

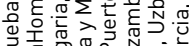

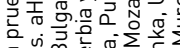

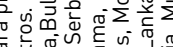

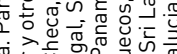

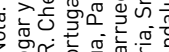


Tabla 2.

Disparidad en las prevalencias de consumo excesivo de alcohol (promedio y episódico) entre nativos españoles e inmigrantes de distintas procedencias

\begin{tabular}{|c|c|c|c|c|c|c|c|c|c|c|c|c|c|c|}
\hline & \multicolumn{7}{|c|}{ Bebedor excesivo promedio } & \multicolumn{7}{|c|}{ Bebedor excesivo episódico } \\
\hline & $\mathrm{N}$ & $\%$ & $\mathrm{RPc}$ & $\mathrm{RPa}^{1,2}$ & $95 \%$ & IC & Valor $p$ & $\mathrm{~N}$ & $\%$ & $\mathrm{RPc}$ & $\mathrm{RPa}^{1}$ & $95 \%$ & IC & Valor $\mathrm{p}$ \\
\hline \multicolumn{15}{|l|}{ Zona de País de Origen } \\
\hline España & 566 & 3,1 & 1 & 1 & & & & 1814 & 10,0 & 1 & 1 & & & \\
\hline Europa del Este & 23 & 4,8 & 1,33 & 1,02 & 0,65 & 1,59 & 0,934 & 52 & 10,4 & 1,00 & $0,74^{*}$ & 0,58 & 0,96 & 0,021 \\
\hline Europa del Norte & 13 & 7 & $2,14^{* *}$ & $2,28^{* *}$ & 1,29 & 4,05 & 0,005 & 31 & 15,0 & 1,30 & $1,81^{* *}$ & 1,25 & 2,62 & 0,002 \\
\hline Europa del Sur & 2 & 1,7 & 0,53 & 0,27 & 0,06 & 1,11 & 0,069 & 6 & 5,0 & 0,51 & $0,15^{* *}$ & 0,05 & 0,46 & 0,001 \\
\hline Europa del Oeste & 15 & 6,9 & $2,16^{* *}$ & $2,12^{* *}$ & 1,29 & 3,49 & 0,003 & 26 & 11,7 & 1,17 & 1,10 & 0,78 & 1,55 & 0,576 \\
\hline América Cono Sur & 6 & 1,7 & 0,6 & 0,55 & 0,26 & 1,16 & 0,115 & 44 & 11,8 & 1,22 & 0,87 & 0,67 & 1,12 & 0,279 \\
\hline América Latina países andinos & 27 & 3,4 & 1,1 & 1,36 & 0,93 & 1,99 & 0,113 & 110 & 13,3 & $1,32^{* *}$ & $1,24^{*}$ & 1,03 & 1,50 & 0,020 \\
\hline Centroamérica y Caribe & 8 & 4,5 & 1,37 & 1,77 & 0,90 & 3,48 & 0,101 & 14 & 7,8 & 0,82 & 1,04 & 0,64 & 1,70 & 0,865 \\
\hline África & 2 & 0,5 & 0,16 & 0,16 & 0,04 & 0,67 & 0,012 & 11 & 2,7 & $0,25^{* *}$ & $0,20^{* *}$ & 0,11 & 0,37 & 0,000 \\
\hline Otros países & 0 & 0 & & N.A & & & N.A & 3 & 2,1 & $0,21^{* *}$ & $0,20^{* *}$ & 0,07 & 0,61 & 0,005 \\
\hline
\end{tabular}

Nota. RPc: Razón de prevalencias Cruda; RPa: Razón de Prevalencias Ajustada. 95\% IC: Intervalo de confianza al 95\%. ${ }^{*}$ p $>0,05,{ }^{* *}$ p<0,01 N.A: No Aplicable Análisis Ajustado por: (1) Sexo, Edad, Zona de residencia, Tamaño de municipio, Convivencia, Nivel de estudios, Situación laboral, Estado de salud percibido, Ansiedad o Depresión, Fuma a diario y consumo de otras sustancias en último año. (2): Exposición a vandalismo o delincuencia.

$(\mathrm{RPa}=0,20 \mathrm{IC} 95 \% 0,11-0,37)$ y Europa del Este $(\mathrm{RPa}=0,74$ IC95\% 0,58-0,96) (Tabla 2).

\section{Factores asociados con el consumo de alcohol en nativos e inmigrantes}

Varios factores se relacionaron con una mayor prevalencia de CEP en nativos e inmigrantes: Tener menos de 34 años y consumir drogas ilegales. Mayor exposición a la violencia, vivir en el centro de España y estar desempleado se asociaron a mayor prevalencia en la población inmigrante, pero no así en la nativa (Tabla 3).

En relación al CEE, se identificaron cuatro factores relacionados con una mayor prevalencia del mismo en ambas poblaciones: ser hombre, tener menos de 35 años, fumar diariamente y consumir otras drogas. Además, solo en los inmigrantes se identificaron como factores relacionados con mayor prevalencia: vivir en poblaciones mayores de 50.000 habitantes frente a las de menos de 10.000, estar desempleado, un buen estado de salud, vivir solo y estar expuesto al vandalismo (Tabla 4).

\section{Discusión}

La prevalencia de consumo excesivo promedio de alcohol es mayor en la población nativa, mientras que la del consumo excesivo episódico es mayor en la inmigrante, si bien ninguna de estas diferencias son significativas. No obstante, existen grandes disparidades dentro de esta población inmigrante si se pormenorizan según el área de procedencia. Así, los inmigrantes procedentes de países europeos, especialmente de Europa Occidental y del Norte, tienen unas prevalencias superiores a las de los autóctonos, mientras que los procedentes de África las tienen menores. Estas disparidades, coincidentes con lo señalado en otros estudios (Adrian, 1996; Conde y Herranz, 2004), persisten al ajustar por posibles factores confusores, por lo que no serían atribuibles simplemente a diferentes patrones sociodemográficos.

Esta heterogeneidad en el consumo dentro de la llamada "población inmigrante" es uno de los principales mensajes del presente estudio. La llegada poco escalonada de inmigrantes a España, ha determinado una imagen de "colectivo" con pocas diferencias en su seno. Lo cual no responde a la realidad y no ayuda a la puesta en marcha de programas de intervención social ajustados a los diferentes subgrupos (Conde y Herranz, 2004). Los perfiles de estas personas varían mucho de unas áreas de procedencia a otra. Desde el perfil de persona mayor, quizá ya jubilada, esencialmente procedente de países europeos, con un buen nivel socioeconómico, que predomina en la costa española, al perfil de inmigrante joven y hombre venido desde países en desarrollo, perteneciente a lo que se ha dado en denominar "inmigrante económico"(OPI, 2006). Es muy posible que estos perfiles tengan su correlato en el consumo de alcohol, por lo que sería necesario confirmar esta hipótesis mediante estudios diseñados ad hoc para ajustar las políticas a las necesidades de los grupos más vulnerables.

Este estudio, además, se centró en los factores relacionados con los patrones de consumo estudiados. Globalmente destaca una escasa coincidencia en los factores asociados al CEP entre inmigrantes y nativos, mientras que el número de factores asociados comunes para el CEE es mucho mayor entre estas dos poblaciones. La mayor vulnerabilidad de los menores de 35 años para ambos patrones es clara tanto en inmigrantes como en nativos. Se trata de la edad en la que más ha aumentado el consumo de alcohol, en especial en forma de CEE (Donath et al., 2011; Valencia-Martin et al., 2007). Por lo que este resultado no hace más que enfatizar la necesidad de priorizar las intervenciones en los jóvenes, tengan la procedencia que tengan.

Además, hay otros aspectos específicos que configuran la vulnerabilidad de la población inmigrante en relación con 
Tabla 3.

Factores asociados al consumo excesivo promedio de alcohol entre inmigrantes y nativos

\begin{tabular}{|c|c|c|c|c|c|c|c|c|c|c|}
\hline & \multicolumn{5}{|c|}{ Inmigrantes } & \multicolumn{5}{|c|}{ Nativos } \\
\hline & $\mathrm{n}$ & $\%$ & $\mathrm{RPc}$ & $\mathrm{RPa}$ & IC95\% & $\mathrm{n}$ & $\%$ & $\mathrm{RPc}$ & $\mathrm{RPa}$ & $\mathrm{IC} 95 \%$ \\
\hline \multicolumn{11}{|l|}{ Sexo } \\
\hline Hombre & 43 & 3,2 & 1 & & & 355 & 4,1 & 1 & & \\
\hline Mujer & 54 & 3,5 & 1,2 & 1,1 & $0,7-1,7$ & 211 & 2,3 & $0,6^{*}$ & $0,8^{*}$ & $0,7-1$ \\
\hline \multicolumn{11}{|l|}{ Edad } \\
\hline $16-34$ & 57 & 3,9 & 1 & & & 210 & 4,2 & 1 & & \\
\hline $35-54$ & 28 & 2,5 & $0,6^{*}$ & $0,5^{*}$ & $0,3-0,9$ & 226 & 3,5 & $0,8^{*}$ & 0,8 & $0,6-1,1$ \\
\hline 55 y más & 12 & 3,8 & 0,9 & 0,9 & $0,5-1,6$ & 131 & 2,1 & $0,4^{*}$ & $0,7^{*}$ & $0,5-1$ \\
\hline \multicolumn{11}{|l|}{ Zona de residencia en España } \\
\hline Sur ${ }^{1}$ & 17 & 2,5 & 1 & & & 160 & 3,5 & 1 & & \\
\hline Este" & 36 & 3,2 & 1,4 & 1,3 & $0,7-2,4$ & 75 & 1,6 & $0,4^{*}$ & $0,4^{*}$ & $0,3-0,6$ \\
\hline Norte"II & 13 & 3,0 & 1,3 & 1,7 & $0,9-3,8$ & 184 & 3,8 & 1,1 & 1,1 & $0,8-1,3$ \\
\hline Centrolv & 30 & 4,7 & $1,9^{*}$ & $2^{*}$ & $1,1-3,9$ & 147 & 4,2 & 1 & 1,0 & $0,8-1,3$ \\
\hline \multicolumn{11}{|l|}{ Tamaño de municipio residencia } \\
\hline$<10000$ habitantes & 14 & 3,6 & 1 & & & 148 & 3,8 & 1 & & \\
\hline $10000-50000$ habitantes & 23 & 2,7 & 0,7 & & & 121 & 2,7 & $0,7^{*}$ & 0,8 & $0,6-1,0$ \\
\hline$>50000$ habitantes & 60 & 3,6 & 1,0 & & & 297 & 3,2 & 0,9 & 0,8 & $0,6-1,0$ \\
\hline \multicolumn{11}{|l|}{ Convivencia } \\
\hline Solo/a & 15 & 6,2 & 1 & & & 61 & 3,8 & 1 & & \\
\hline Otras formas de convivencia & 28 & 3,7 & 0,6 & 0,6 & $0,3-1,1$ & 80 & 4,2 & 1,1 & 1,0 & $0,7-1,4$ \\
\hline Con pareja y/o hijos & 54 & 2,9 & $0,5^{*}$ & 0,8 & $0,4-1,6$ & 426 & 3,0 & $0,8^{*}$ & $0,8^{*}$ & $0,6-1$ \\
\hline \multicolumn{11}{|l|}{ Nivel de estudios } \\
\hline Primarios o menos & 10 & 1,6 & 1 & & & 172 & 2,6 & 1 & & \\
\hline Secundarios (incluido bachiller y profesionales) & 64 & 3,7 & $2,2^{*}$ & 1,4 & $0,7-2,7$ & 290 & 3,7 & $1,5^{*}$ & 1,2 & $1-1,5$ \\
\hline Universitarios & 23 & 4,2 & $2,5^{*}$ & 1,5 & $0,7-3,1$ & 104 & 3,5 & $1,5^{*}$ & $1,4^{*}$ & $1-1,8$ \\
\hline \multicolumn{11}{|l|}{ Situación Laboral } \\
\hline Trabajando & 48 & 3,0 & 1 & & & 319 & 3,8 & 1 & & \\
\hline Desempleado & 37 & 6,0 & $2^{*}$ & $2,2^{*}$ & $1,5-3,3$ & 106 & 5,1 & $1,3^{*}$ & 1,1 & $0,9-1,4$ \\
\hline Otros ${ }^{\beta}$ & 12 & 1,9 & 0,7 & $0,4^{*}$ & $0,2-0,8$ & 137 & 1,9 & $0,5^{*}$ & $0,7^{*}$ & $0,6-0,9$ \\
\hline \multicolumn{11}{|l|}{ Estado de salud percibido ${ }^{\alpha}$} \\
\hline Bueno o muy bueno & 84 & 3,6 & 1 & & & 443 & 3,6 & 1 & & \\
\hline Regular, malo o muy malo & 12 & 2,2 & 0,6 & & & 123 & 2,4 & $0,7^{*}$ & 0,9 & $0,7-1,1$ \\
\hline \multicolumn{11}{|l|}{ Ha padecido ansiedad o depresión ${ }^{\alpha}$} \\
\hline $\mathrm{Si}$ & 3 & 2,0 & 1 & & & 24 & 1,4 & 1 & & \\
\hline No & 93 & 3,4 & 1,7 & & & 543 & 3,4 & $2,4^{*}$ & $1,9 *$ & $1,3-3,0$ \\
\hline \multicolumn{11}{|c|}{ Exposición a delincuencia, violencia o vandalismo ${ }^{\alpha}$} \\
\hline Muy expuesto & 9 & 7,8 & 1 & & & 23 & 3,8 & 1 & & \\
\hline Algo expuesto & 11 & 2,9 & $0,3^{*}$ & $0,4^{*}$ & $0,2-0,5$ & 100 & 3,9 & 0,8 & 0,8 & $0,5-1,3$ \\
\hline No expuesto & 75 & 3,2 & $0,4^{*}$ & 0,5 & $0,2-1,1$ & 443 & 3,1 & $0,7^{*}$ & 0,8 & $0,5-1,2$ \\
\hline \multicolumn{11}{|l|}{ Fuma diariamente } \\
\hline No & 63 & 2,9 & 1 & & & 226 & 1,7 & 1 & & \\
\hline $\mathrm{Si}$ & 33 & 5 & 1,4 & & & 340 & 7 & 5* & $3,1^{*}$ & $2,6-3,8$ \\
\hline \multicolumn{11}{|c|}{ Consumo de cannabis, cocaína, anfetaminas, éxtasis o similares ${ }^{\alpha}$} \\
\hline No & 81 & 3,0 & 1 & & & 409 & 2,5 & 1 & & \\
\hline Sí & 15 & 9,4 & $2,94 *$ & $2,1 *$ & $1,5-3,3$ & 150 & 15,7 & $9,3^{*}$ & $4,2^{*}$ & $3,3-5,2$ \\
\hline
\end{tabular}

Nota. RPc: Razón de Prevalencia cruda; RPa: Razón de Prevalencia ajustada; ${ }^{*} p<0,05 ; \alpha$ En los últimos 12 meses; $\beta$ Estudiantes o personas en formación con prácticas no renumeradas, jubilados o retirados del negocio, incapacitados para trabajar, personas que se dedican principalmente a labores del hogar y otros. I Andalucía, Murcia, Canarias, Ceuta-Melilla; II Valencia, Baleares, Cataluña; III Aragón, Rioja, Navarra, País Vasco, Cantabria, Asturias, Galicia, Castilla-León; IV Madrid, Castilla-La Mancha, Extremadura.

el consumo de alcohol, y que han de tenerse en consideración: 1) Como se había visto en otros estudios (Ministerio de Sanidad, Servicios Sociales e Igualdad, 2013), los inmigrantes que viven en la zona centro de España constituyen un grupo diana para mensajes orientados a la prevención del CEP. 2) Entre los inmigrantes, el desempleo se asocia a un mayor consumo para ambos patrones, aunque la fuerza de la asociación es superior en el CEP. La razón mayoritaria para inmigrar suele ser trabajar (Skarlund, Ahs, y Westerling, 2012), y el impacto de su presencia o ausencia parece influir notablemente en el consumo de alcohol en esta población, como ya había sido indicado (So y Wong, 2006). La relación entre deterioro de salud, consumo de alcohol y desempleo es multidireccional. Cada uno de estos factores pueden ser causa o consecuencia de los demás (Skarlund et al.,
2012). Y si esto ocurre en cualquier población, es especialmente importante en la inmigrante, cuya situación laboral y redes de apoyo suelen ser más inestables (Gutmann, 1999). 3) En los no nativos, la exposición a la violencia se asocia a un mayor consumo de alcohol tanto para el CEP como el CEE. Cabe preguntarse nuevamente si este factor es causa o efecto, pero puede dar a la interpretación de una mayor vulnerabilidad. 4) La prevalencia de CEE es superior entre los inmigrantes que residen en ciudades grandes. Quizá se deba a que es en estas donde los inmigrantes encuentran entornos con más personas de su misma procedencia, lo cual podría aumentar su red social y en consecuencia, su CEE. En cualquier caso, sí lleva a hacer una reflexión sobre si las alternativas de ocio que se ofrecen en España, pudieran no encajar con las demandas de la población inmigrante. 
Tabla 4.

Factores asociados al consumo excesivo episódico de alcohol entre inmigrantes y nativos

\begin{tabular}{|c|c|c|c|c|c|c|c|c|c|c|}
\hline & \multicolumn{5}{|c|}{ Inmigrantes } & \multicolumn{5}{|c|}{ Nativos } \\
\hline & $\mathrm{n}$ & $\%$ & $\mathrm{RPc}$ & $\mathrm{RPa}$ & IC95\% & $\mathrm{n}$ & $\%$ & $\mathrm{RPc}$ & $\mathrm{RPa}$ & IC95\% \\
\hline \multicolumn{11}{|l|}{ Sexo } \\
\hline Hombre & 194 & 13,7 & 1 & & & 1405 & 15,7 & 1 & & \\
\hline Mujer & 102 & 6,5 & $0,4^{*}$ & $0,5^{*}$ & $0,4-0,6$ & 408 & 4,4 & $0,2^{*}$ & $0,3^{*}$ & $0,3-0,4$ \\
\hline \multicolumn{11}{|l|}{ Edad } \\
\hline $16-34$ & 176 & 11,8 & 1 & & & 957 & 18,0 & 1 & & \\
\hline $35-54$ & 106 & 9,1 & $0,8^{*}$ & $0,8^{*}$ & $0,6-0,9$ & 684 & 10,2 & $0,5^{*}$ & $0,7^{*}$ & $0,6-0,8$ \\
\hline 55 y más & 15 & 4,6 & $0,4^{*}$ & $0,3^{*}$ & $0,2-0,6$ & 173 & 2,7 & $0,1^{*}$ & $0,3^{*}$ & $0,2-0,3$ \\
\hline \multicolumn{11}{|l|}{ Zona de residencia en España } \\
\hline Sur' & 66 & 9,4 & 1 & & & 462 & 10,0 & 1 & & \\
\hline Este" & 107 & 9,1 & 0,9 & 1,0 & $0,8-1,6$ & 339 & 6,8 & $0,7^{*}$ & $0,7^{*}$ & $0,6-0,8$ \\
\hline Norte"II & 37 & 8,4 & 0,9 & 0,9 & $0,7-1,5$ & 528 & 10,8 & 1,1 & $1,2^{*}$ & $1-1,3$ \\
\hline Centroiv & 87 & 13,0 & $1,4^{*}$ & 1,1 & $0,8-1,6$ & 485 & 13,5 & $1,4^{*}$ & $1,3^{*}$ & $1,1-1,5$ \\
\hline \multicolumn{11}{|l|}{ Tamaño de municipio residencia } \\
\hline$<10000$ habitantes & 24 & 6,1 & 1 & & & 398 & 10,1 & 1 & & \\
\hline $10000-50000$ habitantes & 78 & 9,0 & 1,4 & 1,3 & $0,8-2,1$ & 430 & 9,3 & 0,9 & & \\
\hline$>50000$ habitantes & 195 & 11,3 & $1,8^{*}$ & $1,6^{*}$ & $1,1-2,5$ & 986 & 10,3 & 1,0 & & \\
\hline \multicolumn{11}{|l|}{ Convivencia } \\
\hline Solo & 35 & 13,8 & 1 & & & 143 & 8,7 & 1 & & \\
\hline Otras formas de convivencia & 92 & 11,9 & 0,9 & 0,9 & $0,6-1,3$ & 1464 & 10,1 & $1,2^{*}$ & 0,9 & $0,8-1,1$ \\
\hline Con pareja y/o hijos & 169 & 8,6 & $0,7^{*}$ & $0,7^{*}$ & $0,5-0,9$ & 207 & 10,5 & $1,2^{*}$ & 1,0 & $0,8-1,3$ \\
\hline \multicolumn{11}{|l|}{ Nivel de estudios } \\
\hline Primarios o menos & 34 & 5,4 & 1 & & & 369 & 5,4 & 1 & & \\
\hline Secundarios (incluido bachiller y profesionales) & 206 & 11,6 & $2,2^{*}$ & 1,4 & $0,9-2$ & 1045 & 12,7 & $2,4^{*}$ & $1,4^{*}$ & $1,2-1,6$ \\
\hline Universitarios & 54 & 9,6 & $1,8^{*}$ & 1,3 & $0,8-2$ & 400 & 12,8 & $2,5^{*}$ & $1,8^{*}$ & $1,5-2,0$ \\
\hline \multicolumn{11}{|l|}{ Situación Laboral } \\
\hline Trabajando & 153 & 9,2 & 1 & & & 1099 & 12,7 & 1 & & \\
\hline Desempleado & 88 & 14,0 & $1,5^{*}$ & $1,4^{*}$ & $1,1-1,8$ & 302 & 14,0 & 1,1 & 1,0 & $0,8-1,1$ \\
\hline Otros ${ }^{\beta}$ & 54 & 8,0 & 0,8 & 1,1 & $0,8-1,7$ & 400 & 5,5 & $0,4^{*}$ & 0,9 & $0,8-1,1$ \\
\hline \multicolumn{11}{|l|}{ Estado de salud percibido ${ }^{\alpha}$} \\
\hline Bueno o muy bueno & 252 & 10,4 & 1 & & & 1535 & 11,9 & 1 & & \\
\hline Regular, malo o muy malo & 44 & 7,7 & $0,6^{*}$ & $0,7^{*}$ & $0,5-0,9$ & 279 & 5,3 & $0,4^{*}$ & 0,9 & $0,8-1$ \\
\hline \multicolumn{11}{|l|}{ Ha padecido ansiedad o depresión ${ }^{\alpha}$} \\
\hline $\mathrm{Si}$ & 10 & 6,4 & 1 & & & 72 & 4,1 & 1 & & \\
\hline No & 286 & 10,1 & 1,6 & & & 1742 & 10,6 & $2,8^{*}$ & $1,4^{*}$ & $1,1-1,9$ \\
\hline \multicolumn{11}{|c|}{ Exposición a delincuencia, violencia o vandalismo ${ }^{\alpha}$} \\
\hline Muy expuesto & 21 & 17,6 & 1 & & & 52 & 8,4 & 1 & & \\
\hline Algo expuesto & 41 & 10,1 & $0,5^{*}$ & 0,6 & $0,3-1$ & 301 & 11,2 & 1,2 & & \\
\hline No expuesto & 233 & 9,5 & $0,5^{*}$ & $0,6^{*}$ & $0,3-0,9$ & 1461 & 9,9 & 1,3 & & \\
\hline \multicolumn{11}{|l|}{ Fuma diariamente } \\
\hline Sí & 121 & 16,1 & 1 & & & 865 & 18,2 & & & \\
\hline No & 176 & 7,9 & $0,5^{*}$ & $0,5^{*}$ & $0,4-0,7$ & 944 & 7,1 & $0,4^{*}$ & $0,6^{*}$ & $0,5-0,7$ \\
\hline \multicolumn{11}{|c|}{ Consumo de cannabis, cocaína, anfetaminas, éxtasis o similares ${ }^{\alpha}$} \\
\hline No & 249 & 9,1 & 1 & & & 1360 & 8,1 & 1 & & \\
\hline Sí & 45 & 26,2 & $2,9 *$ & $2^{*}$ & $1,4-2,7$ & 432 & 42,6 & $5,1^{*}$ & $2,4^{*}$ & $2,1-2,7$ \\
\hline
\end{tabular}

Nota. cRP: Razón de Prevalencia cruda; RPa: Razón de Prevalencia ajustada; ${ }^{*} p<0,05 ; \alpha$ En los últimos 12 meses; $\beta$ Estudiantes o personas en formación con prácticas no renumeradas, jubilados o retirados del negocio, incapacitados para trabajar, personas que se dedican principalmente a labores del hogar y otros. I Andalucia, Murcia, Canarias, Ceuta-Melilla; II Valencia, Baleares, Cataluña; III Aragón, Rioja, Navarra, País Vasco, Cantabria, Asturias, Galicia, Castilla-León; IV Madrid, Castilla-La Mancha, Extremadura

Los inmigrantes tienen diferentes pautas de comportamiento, valores y percepciones respecto al consumo de drogas relacionadas con las vigentes en sus países de origen (Daniel-Ulloa et al., 2014). El proceso de migración produce nuevas situaciones y oportunidades (Alaniz, 2002). El consumo de sustancias no es ajeno al proceso de aculturación o adaptación a la cultura del país receptor (Zemore, 2007). Y en este proceso influye tanto la cultura del país de origen como la del de acogida, en este caso España. En este sentido convendría, como se ha hecho en otros países (Delva et al., 2005; Sussman, 2005), que las intervenciones encontraran el equilibrio entre el reforzamiento positivo de las conductas protectoras "de origen" y la identificación de aquellos procesos y situaciones que puedan aumentar el riesgo de consumir alcohol de forma excesiva. Además, el consumo de alcohol no puede analizarse como un hecho aislado. Guarda una estrecha relación con los procesos generales de integración. Hasta el punto que muchos autores lo analizan en un contexto más amplio y como síntoma o indicador de procesos de integración (Condey Herranz, 2004). La investigación en esta población debe mantenerse en el tiempo. La gran llegada de inmigrantes a España ha cesado en los últimos años, pero la mayoría de los que llegaron, lo hicieron para quedarse (INE, 2013).

Como limitaciones al estudio cabe redundar en que su diseño transversal no permite conocer la dirección de la asociación ni establecer causalidad. En lo que se refiere a la variable de lugar de origen, no sabemos el tiempo que hace que llegaron a España. Ha sido descrito que los patrones de consumo de alcohol en población inmigrante se van acer- 
cando a los de la población autóctona conforme transcurren los años viviendo en el país de acogida (De La Rosa et al., 2012). Se trata de una variable que no está contemplada en la encuesta y que debe tenerse en cuenta para sucesivas. No obstante, la gran mayoría de los inmigrantes que había en España en 2010, fecha de finalización de esta encuesta, habían llegado a España en los años inmediatamente anteriores (INE, 2013), por lo que quizá los resultados no fueran muy diferentes. En este sentido, algunos estudios similares a este realizados en otros entornos no encontraron diferencias en función del tiempo de permanencia en el país de acogida(Szaflarski, Cubbins, y Ying, 2011). Otra limitación, fue la imposibilidad de dar los resultados en función de países y no de zonas. A pesar del gran tamaño muestral, este es insuficiente para un análisis "país por país". Sería recomendable que las áreas señaladas en el presente estudio como aquellas con mayor consumo de alcohol (Europa del norte y del oeste, y América latina en el consumo excesivo episódico) fueran susceptibles de investigaciones pormenorizadas para no caer en tratar a estas poblaciones como "colectivos". Incluso empleando esta variable en función de zonas y no países, algunas aportan tan poca muestra que los resultados relativos a estas han de interpretarse con mucha cautela.

Otro aspecto deficitario es la recogida de otras variables de consumo de sustancias. Son poco profundas y no permitieron análisis más elaborados que los que se presentan para determinar su relación con el consumo de alcohol (este sí muy bien recogido y pormenorizado). Se trata de uno de los problemas derivados de este tipo de amplias encuestas poblacionales que a cambio nos dan un enorme tamaño muestral. Y finalmente, las personas sin hogar, por la estrategia de recogida de datos, no se incluyeron en el estudio. Población que en lo referido a consumo de alcohol puede ser especialmente relevante.

Más allá de las limitaciones, el presente estudio proporciona una primera aproximación a la epidemiología del consumo de alcohol en la población inmigrante que vive en España, al tiempo que identifica subgrupos sobre los que quizá fuera conveniente realizar intervenciones concretas. En especial en lo que se refiere al consumo excesivo episódico. Para planificar posibles estrategias preventivas de presente y de futuro. El aumento de la población inmigrante en España demanda un abordaje integral de sus problemas de salud y, en este contexto, el consumo excesivo de alcohol es prioritario.

\section{Reconocimientos}

\section{Financiación}

Los autores agradecen la ayuda económica del Plan Nacional sobre Drogas (órdenes SSI 2800/2012 y 131/2013) y de la Agencia Estratégica en Salud (AES) (PI13/00183 y PI
11/01396.). La elaboración de este trabajo ha contado también con la financiación de la Red de Trastornos Adictivos (RTA) (RD12/0028/0018) y de los proyectos 2011/059. La elaboración del manuscrito ha recibido apoyo a través del contrato (CD10/00128 -Sara Borrell)

\section{Conflicto de intereses}

Los autores declaran no tener ningún conflicto de intereses.

\section{Referencias}

Adrian, M. (1996).Substance use and multiculturalism.Substance Use \& Misuse, 31, 1459-1501.

Alaniz, M. L. (2002). Migration, acculturation, displacement: migratory workers and "substance abuse". Substance Use \& Misuse, 37, 1253-1257.

Anderson P, M. (2012). Alcohol in the European Union. Consumption, harm and policy approaches. World Health Organization.Regional Office for Europe.

Babor, T. F., McRee, B. G., Kassebaum, P. A., Grimaldi, P. L., Ahmed, K. y Bray, J. (2007). Screening, Brief Intervention, and Referral to Treatment (SBIRT): toward a public health approach to the management of substance abuse. Substance Abuse, 28, 7-30.

Carrasco-Garrido, P., de Miguel, A. G., Barrera, V. H. y Jimenez-Garcia, R. (2007). Health profiles, lifestyles and use of health resources by the immigrant population resident in Spain. European Journal of Public Health, 17, 503-507.

Conde, F. y Herranz D. (2004). Los procesos de integración de los inmigrantes. Pautas de consumo de alcohol y modelos culturales de referencia. Madrid: Fundación CREFAT.

Daniel-Ulloa, J., Reboussin, B. A., Gilbert, P. A., Mann, L., Alonzo, J., Downs, M., y Rhodes, S. D. (2014). Predictors of heavy episodic drinking and weekly drunkenness among immigrant latinos in North Carolina. American Journal of Men's Health, 8, 339-348.

De La Rosa, M., Sanchez, M., Dillon, F. R., Ruffin, B. A., Blackson, T. y Schwartz, S. (2012). Alcohol use among Latinos: a comparison of pre-immigration, post-immigration, and US born Latinos. Journal of Immigrant and Minority Health, 14, 371-378.

De Maio, F. G. (2010). Immigration as pathogenic: a systematic review of the health of immigrants to Canada. International Journal for Equity in Health, 9, 27.

Delva, J., Wallace, J. M., Jr., O’Malley, P. M., Bachman, J. G., Johnston, L. D. y Schulenberg, J. E. (2005). The epidemiology of alcohol, marijuana, and cocaine use among Mexican American, Puerto Rican, Cuban American, and other Latin American eighth-grade students in the United States: 1991-2002. American Journal of Public Health, 95, 696-702. 
Donath, C., Grassel, E., Baier, D., Pfeiffer, C., Karagulle, D., Bleich, S., y Hilllemacher, T. (2011). Alcohol consumption and binge drinking in adolescents: comparison of different migration backgrounds and rural vs. urban residence: a representative study. BMC Public Health, 11, 84.

Gutmann, M. C. (1999). Ethnicity, alcohol, and acculturation. Social Science E Medicine, 48, 173-184.

Instituto Nacional de Estadística (INE). (2009). Encuesta Europea de Salud en España EES-2009. Metodología. Madrid: Ministerio de Sanidad, Politica Social e Igualdad.

Instituto Nacional de Estadística (INE). (2013). Cifras de Población y Estadística de Migraciones. Madrid: Ministerio de Sanidad, Politica Social e Igualdad.

Lim, S. S., Vos, T., Flaxman, A. D., Danaei, G., Shibuya, K., Adair-Rohani, H., ... Ezzati, M. (2012). A comparative risk assessment of burden of disease and injury attributable to 67 risk factors and risk factor clusters in 21 regions, 1990-2010: a systematic analysis for the Global Burden of Disease Study 2010. Lancet, 380, 2224-2260.

MacPherson, D. W. y Gushulak, B. D. (2001). Human mobility and population health. New approaches in a globalizing world. Perspectives in Biology and Medicine, 44,390-401.

Marsiglia, F. F., Kulis, S., Luengo, M. A., Nieri, T. y Villar, P. (2008). Immigrant advantage? Substance use among Latin American immigrant and native-born youth in Spain. Ethnicity and Health, 13, 149-170.

Martinez-Hernaez, A., Mari-Klose, M., Julia, A., Escapa, S. y Mari-Klose, P. (2012). Heavy episodic drinking among adolescents: the association with negative mood states and family factors. Revista Española de Salud Pública, 86, 101-114.

Mills, B. A. y Caetano, R. (2012). Decomposing associations between acculturation and drinking in Mexican Americans. Alcoholism Clinical and Experimental Research, 36, 1205-1211.

Ministerio de Sanidad, Servicios Sociales e Igualdad (MSSSI). (2013). Serie Informes monográficos $n^{\circ} 1$. Consumo de alcohol. Madrid: MSSSI.

Observatorio permanente de la inmigración. (2006). Extranjeros con tarjeta o autorización de residencia en vigor a 31 de marzo de 2006. Madrid: Ministerio de Trabajo y Asuntos Sociales.

Rehm, J., Rehm, M. X., Shield, K. D., Gmel, G. y Gual, A. (2013). Consumo de alcohol, dependencia alcohólica, trastornos relacionados con el alcohol en España. Impacto de los tratamientos de la dependencia alcohólica. Adicciones, 25, 11-18.

Skarlund, M., Ahs, A. y Westerling, R. (2012).Health-related and social factors predicting non-reemployment amongst newly unemployed.BMC Public Health, 12, 893.

Schmidt, L.A, Mäkelä, P, Rehm, J, Room, R. Alcohol: equity and social determinants. En: Blass E, Kurup, A.S, editors. Equity, social determinants and public health programmes. (2010). Geneva: World Health Organization.

So, D. W. y Wong, F. Y. (2006). Alcohol, drugs, and substance use among Asian-American college students. Journal of Psychoactive Drugs, 38, 35-42.

Spiegelman, D. y Hertzmark, E. (2005).Easy SAS calculations for risk or prevalence ratios and differences.American Journal of Epidemiology, 162, 199-200.

Sussman, S. (2005).Prevention of adolescent alcohol problems in special populations. Recent Developments in Alcoholism, 17, 225-253.

Szaflarski, M., Cubbins, L. A. y Ying, J. (2011). Epidemiology of alcohol abuse among US immigrant populations. Journal of Immigrant and Minority Health, 13, 647-658.

Valencia-Martin, J. L., Galan, I. y Rodriguez-Artalejo, F. (2007). Binge drinking in Madrid, Spain.Alcoholism Clinical and Experimental Research, 31, 1723-1730.

World Health Organization (WHO).(2000). International Guide for Monitoring Alcohol Consumption and Related Harm. Geneva: WHO.

World Health Organization (WHO). (2006). International Standard ISO 3166-1, Codes for the representation of names of countries and their subdivisions-Part 1: Country codes, ISO 3166-1: 2006. International Organization on Standardization. Geneva: WHO.

World Health Organization (WHO). (2014). The global status report on alcohol and health 2014. Geneva: WHO.

Zamboanga, B. L., Schwartz, S. J., Jarvis, L. H., y Van, T. K. (2009). Acculturation and substance use among Hispanic early adolescents: investigating the mediating roles of acculturative stress and self-esteem. The Journal of Primary Prevention, 30,315-333.

Zemore, S. E. (2007). Acculturation and alcohol among Latino adults in the United States: a comprehensive review. Alcoholism Clinical and Experimental Research, 31, 1968-1990. 